\title{
Single Pulse Multiphoton Fabrication of Photopolymerized Periodic Structures Using Vortex Beams
}

\author{
B. Mills ${ }^{1}$, D. Kundys ${ }^{1}$, M. Farsari ${ }^{2}$, S. Mailis ${ }^{1}$, R. W. Eason ${ }^{1}$ \\ ${ }^{1}$ Optoelectronics Research Centre, University of Southampton, Southampton, UK, SO17 1BJ \\ ${ }^{2}$ IESL-FORTH, Heraklion, Greece \\ Author (B. Mills) e-mail address: bm602@orc.soton.ac.uk, Tel: (+44) 0238059 4761, Fax: (+44) 02380593142
}

\begin{abstract}
Single ultra-short pulses with a vortex phase profile have been used to fabricate periodically modulated hollow cylinders via multi-photon polymerization. Fabrication and spectral characterization of these objects are presented.

OCIS codes: (020.4180) Multiphoton processes; (260.3160) Interference;
\end{abstract}

\section{Introduction}

Multiphoton polymerization by ultrafast lasers is a technique that enables the fabrication of complex threedimensional objects that can have resolution below the optical diffraction limit [1]. By careful choice of the laser intensity with respect to the multiphoton polymerization threshold, spatial resolution better than $100 \mathrm{~nm}$ has been achieved [2]. Applications for these structures have been proposed in a wide variety of fields such as photonics [3], metamaterials [4] and biomedical devices [5].

Multiphoton polymerization typically involves scanning a focused laser beam in three dimensions with respect to the sample to produce a solid 3D object. Here however, we show that the use of specialty beam intensity profiles in conjunction with optical interference can bypass the requirements for beam scanning to produce complex photopolymerized structures. In this work we present micro-fabrication of objects with superimposed ultrafine periodic features with hollow cores that would be difficult and time consuming to fabricate through use of a scanning Gaussian beam profile. We present experimental results for a laser irradiation procedure that uses a single ultrashort laser pulse of $\sim \mu \mathrm{J}$ energy level to create $\sim 5 \mu \mathrm{m}$ high periodic structures that support features down to $\sim 100$ $\mathrm{nm}$. These features are created through the interference of a vortex beam with its own reflection from the sample surface.

\section{Experimental}

For the irradiation process, single $150 \mathrm{fs}$ pulses with a centre wavelength of $800 \mathrm{~nm}$ from a Coherent RegA ultrafast laser amplifier, were focused to a spot size of $\sim 1 \mu \mathrm{m}$ using a 50x infinity corrected objective, inside a polymer of thickness 5-10 $\mu \mathrm{m}$ that had been spin-coated on a $150 \mu \mathrm{m}$ thick microscope cover-slip. The vortex phase plate that was used (RPC Photonics) corresponded to a phase shift of $4 \pi$ over a full revolution around the beam's axis (charge two). After laser exposure, the non-photopolymerized material was removed through submersion in 1propanol. The photopolymer used was an organic-inorganic hybrid material containing silicon and zirconium alkoxides, whose preparation has been described previously [6].

Given that the reflection at the glass-polymer interface is observed to be insignificant due to index matching between glass and polymer at $800 \mathrm{~nm}$, we estimate an intensity reflection coefficient of $4 \%$ at the polymer-air interface, resulting in an intensity profile, inside the polymer, that has a modulation depth of $2 \sqrt{ }\left(I_{1} I_{2}\right) /\left(I_{1}+I_{2}\right) \sim 38 \%$, where $\mathrm{I}_{1}, \mathrm{I}_{2}$ are the incident and reflected intensities respectively. The probability of multiphoton polymerisation occurring, and hence the profile of the structure that is produced, corresponds to this modulated intensity profile. The interference fringes are observed to have good visibility because the path length, which depends on the thickness of the polymer, does not change in the duration of a single pulse. Although the vortex beam has a spiral phase, upon reflection at the sample-air interface the helicity of the phase along the original propagation direction of the beam does not change, and hence the resultant interference fringes are identical to those produced from two overlapped Gaussian beams, except for the phase anomaly in the centre of the beam, which results in a region of zero intensity.

Figure 1a) shows an SEM image of a close-up of a large array of such structures, which can be easily fabricated in great numbers at $250 \mathrm{kHz}$, which is the repetition rate of RegA. The pulse energy in each case is $0.8 \mu \mathrm{J}$. Worthy 
of note is the highly repeatable nature of this rapid fabrication technique. The SEM image reveals an average period of $\sim 200 \mathrm{~nm}$ for these structures. Some aberrations of the polymerized fringes can be seen occasionally in some of the structures, which can be attributed to the action of surface tension of the solvent when evaporating at the development stage following irradiation. A critical point dryer could however eliminate this problem.

The spectral behavior of these periodic structures was investigated using a micro spectrophotometer. These measurements revealed prominent spectral features in both reflection and transmission. Figure 1b) shows a welldefined transmission dip and a corresponding reflection peak centered at $500 \mathrm{~nm}$, which corresponds to the expected spectral response of the grating structure.
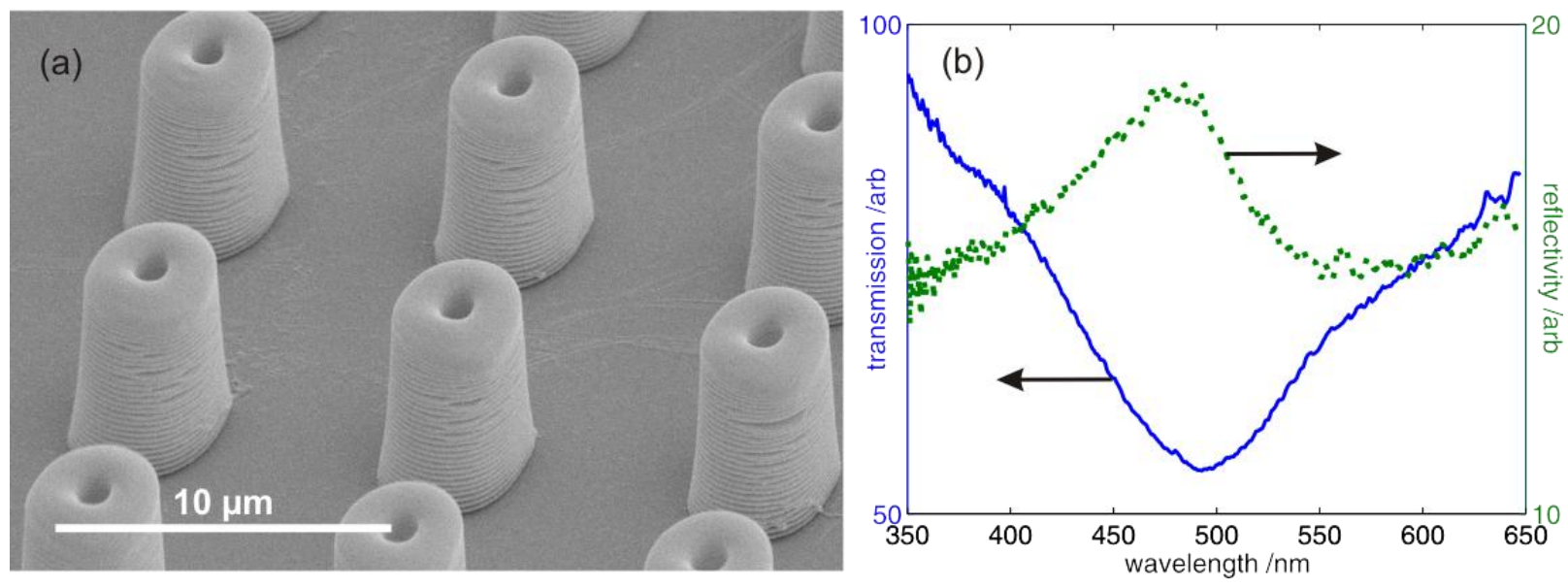

Fig. 1. a) SEM image of an array of modulated structures and b) associated transmission and reflectivity curves.

In conclusion we have presented the fabrication and characterization of optical microstructures with a complex spatial profile using vortex beams and single pulse multiphoton polymerization. These micro structures show potential for the development of metamaterials, for example in 'Light Well' applications, which utilize a focused electron beam injected into such spatially modulated micro-devices [7].

\section{References}

[1] G. vonFreymann, A. Ledermann, M. Thiel, I. Staude, S. Essig, K. Busch, and M. Wegener, "Three-Dimensional Nanostructures for Photonics," Adv. Funct. Mater. 20, 1038-1052 (2010).

[2] W. Haske, V. W. Chen, J. M. Hales, W. T. Dong, S. Barlow, S. R. Marder, and J. W. Perry, "65 nm feature sizes using visible wavelength 3-D multiphoton lithography," Opt. Express 15, 3426-3436 (2007).

[3] T. Grossmann, S. Schleede, M. Hauser, T. Beck, M. Thiel, G. von Freymann, T. Mappes, and H. Kalt, "Direct laser writing for active and passive high-Q polymer microdisks on silicon," Opt. Express 19, 11451-11456 (2011).

[4] J. K. Gansel, M. Thiel, M. S. Rill, M. Decker, K. Bade, V. Saile, G. von Freymann, S. Linden, and M. Wegener, "Gold Helix Photonic Metamaterial as Broadband Circular Polarizer," Science 325, 1513-1515 (2009).

[5] S. D. Gittard, and R. Narayan, "Laser direct writing of micro- and nano-scale medical devices," Expert Rev. Med. Devices 7, 343-356 (2010).

[6] A. Ovsianikov, J. Viertl, B. Chichkov, M. Oubaha, B. MacCraith, L. Sakellari, A. Giakoumaki, D. Gray, M. Vamvakaki, M. Farsari, and C. Fotakis, "Ultra-Low Shrinkage Hybrid Photosensitive Material for Two-Photon Polymerization Microfabrication," ACS Nano 2, $2257-2262$ (2008).

[7] G. Adamo, K. F. MacDonald, Y. H. Fu, C-M. Wang, D. P. Tsai, F. J. Garci'a de Abajo, and N. I. Zheludev, "LightWell: A Tunable FreeElectron Light Source on a Chip", PRL 103, 113901 (2009) 